\title{
Struktur und Funktion der Haut: Physiologie
}

J. Welzel

\section{Physiologie der Haut}

Die Haut ist die Grenze des Körpers zur Umgebung. Die Physiologie beschäftigt sich mit den Funktionen, die das Hautorgan im Kontakt mit äußeren Einflüssen ausübt. Auf diese Funktionen sowie die relevanten Strukturelemente, die diese beeinflussen, wird im folgenden näher eingegangen.

\section{Funktionen}

Die Haut hat eine Schutzfunktion gegenüber äußeren Einflüssen, sie dient dem Austausch von Wärme und Substanzen und der Wahrnehmung äußerer Reize.

$\mathrm{Zu}$ den Barrierefunktionen der Haut gehört der physikalische Schutz gegenüber mechanischen Kräften wie Zug, Druck, Stoß und Scherkräften. Er wird durch elastische Verschiebeschichten wie das Stratum lucidum, Strukturelementen wie das Zytoskelett der Epidermis und das Kollagengeflecht der Dermis sowie Verzahnungen der Retezapfen gewährleistet. Das subkutane Fettgewebe dient mit dem läppchenartigen Aufbau und den Bindegewebssepten als elastisches Polster. Des weiteren schützt die Haut den Körper durch ihren Aufbau vor Austrocknung und in Form der Lichtschwiele und Melaninpigmentierung vor Strahleneinwirkungen.

Dem Schutz gegenüber chemischen Substanzen dient der Hydrolipidfilm der Hautoberfläche, der epidermale Lipide und Enzyme enthält und durch die Produktion der Schweiß- und Talgdrüsen aufrechterhalten wird. Er bietet durch den sogenannten Säureschutzmantel eine Pufferkapazität. Der backsteinmauerartige Aufbau der Hornschicht stellt ebenfalls eine Barriere für chemische Substanzen dar.

Eine biologische Barriere gegenüber Mikroorganismen wird durch den sauren pH-Wert der Hautoberfläche und durch die symbiotische Hautflora hergestellt, dazu kommen immunologische Abwehrmechanismen der Epidermis und Dermis.

Die wichtigste Austauschfunktion der Haut ist die Temperaturregulation. Der Wärmeabstrom durch Verdunstung von Schweiß und Wasser, die durch die Haut diffundieren, steht dabei vor der trockenen Wärmeabgabe oder -aufnahme durch Leitung, Konvektion und Strahlung im Vordergrund. Neben passiven Mechanismen der Haut zur thermischen Isolierung beeinflussen aktive Prozesse wie Durchblutung, Schwitzen und Muskelarbeit die Temperaturregulation. Die Regulierung ist an Rezeptoren gebunden.

Der Stoffaustausch in Form perkutaner Absorption hauptsächlich gasförmiger Substanzen geschieht über Diffusion, spielt aber beim Menschen keine wesentliche Rolle. Eine Bedeutung hat lediglich die perkutane Resorption großflächig aufgetrage- 
ner Substanzen, insbesondere lipophiler Stoffe. Diese kann über die Epidermis, die Haarfollikel und die Schweißdrüsen erfolgen.

Eine weitere Funktion der Haut ist die Reizaufnahme. Sinnesempfindungen wie Tastsinn, Temperatursinn und Schmerzsinn werden über Rezeptoren wahrgenommen und weitergeleitet. Eine besondere Sinnesempfindung der Haut ist der Juckreiz.

\section{Strukturen}

Die Funktionen werden von wichtigen Strukturelementen der Haut beeinflußt. Dazu gehört die Hautoberfläche, deren Größe durch die Rauhigkeit determiniert wird und Faktoren wie Wasser- und Wärmetransport und Resorption beeinflußt. Die Integrität des Aufbaus des Stratum corneum hat eine wesentliche Bedeutung für die Barrierefunktionen der Haut. Die Dicke der Hornschicht sowie die Art der Verhornung (Parakeratose, Schuppung) spielen dabei eine große Rolle. Die Dicke der lebenden Epidermis und Faktoren wie Proliferation, Differenzierung und Spongiose beeinflussen mechanische Parameter ebenso wie Abwehrfunktion und Penetration. Auch bei der Dermis wirken sich Strukturelemente wie Dicke, Durchblutung, Ödem und Infiltrate auf verschiedene Funktionen (z.B. mechanischer Schutz, Temperaturregulation) aus.

\section{Hautphysiologische Meßmethoden}

Es gibt zahlreiche Methoden zur Untersuchung der Hautfunktionen. Eine grobe Orientierung läßt sich durch einfache funktionelle Tests erzielen. Die Kriterien, die Meßverfahren erfüllen sollten, sind vielfältig. Zunächst sollten sie am Menschen durchführbar sein, um relevante Ergebnisse zu erzielen und Tierversuche zu vermeiden. Dafür sind nicht-invasive Methoden vorzuziehen, die Verlaufsbeobachtungen zulassen. Die Methode sollte möglichst objektiv, d.h. vom Untersucher und von Umgebungsbedingungen weitgehend unabhängig sein und eine Quantifikation ermöglichen. Variable Parameter sind zu standardisieren. Dies betrifft insbesondere die sehr empfindlichen Messungen des transepidermalen Wasserverlustes, die in einem klimatisierten Raum nach Akklimatisation an ruhenden Probanden durchgeführt werden müssen, um verwertbare Ergebnisse zu erhalten [6]. Generell sollten die Messungen schnell durchführbar und praktikabel sein und zuverlässige, reproduzierbare Resultate liefern.

\section{Übersicht}

Funktionelle Meßmethoden lassen sich von rein morphologischen unterscheiden, wobei Daten über die Hautfunktion immer auch strukturelle Elemente widerspiegeln. Wichtige hautphysiologische Meßmethoden sind Untersuchungsverfahren des transepidermalen Wasserverlustes, der elektrischen Kapazität, des pH-Wertes, des Fettgehaltes, der Elastizität, der Hautfarbe, der Durchblutung, der Hautoberflächenrauhigkeit und der Morphologie von Hornschicht, Epidermis und Dermis. 


\section{Messungen der Hautfunktionen}

$\mathrm{Zu}$ den wichtigsten Instrumenten zur Ermittlung der Integrität der Barrierefunktion gehört die Messung des transepidermalen Wasserverlustes. Die Hydratation oberflächlicher Hautschichten, die ein wichtiger Parameter für das äußere Erscheinungsbild der Haut ist, läßt sich mit verschiedenen Methoden ermitteln, von denen hier auf die Messung der elektrischen Kapazität exemplarisch näher eingegangen wird. Zur Untersuchung des Säureschutzmantels kann der pH-Wert der Hautoberfläche mit pH-Metern bestimmt werden. Die Elastizität, die insbesondere bei Untersuchungen zur Hautalterung interessant ist, wird quantifiziert, indem eine Zug- oder Drehkraft auf die Haut ausgeübt wird, und das Ausmaß der Auslenkung optisch oder sonographisch erfaßt wird. Objektive Farbmeßinstrumente liefern Daten über entzündliche Veränderungen oder Bräunung. Das Oberflächenfett läßt sich über die sog. Fettfleckphotometrie quantifizieren [11]. Daten über die Hautdurchblutung liefern LaserDoppler-Perfusionsmeßgeräte. Im folgenden wird auf einige dieser Methoden, die für Testungen von Kosmetika und Hautpflegeprodukten besonders relevant sind, näher eingegangen.

\section{Transepidermaler Wasserverlust}

Der transepidermale Wasserverlust der Haut ist ein Maß für die Integrität der Barrierefunktion der Hornschicht.

Bei der Messung wird ein Hohlzylinder auf die Hautoberfläche aufgesetzt. In diesem befinden sich in verschiedener Höhe Sensoren für Wasserdampfdruck und Temperatur. Der Gradient zwischen den Sensorpaaren wird errechnet und spiegelt die Verdunstungsrate in $\mathrm{g} / \mathrm{m}^{2} \mathrm{~h}$ wieder [4].

Bei Störungen der Barrierefunktion, wie sie durch Herauslösen der interzellulären Lipidschichten des Stratum corneum oder entzündliche Veränderungen hervorgerufen werden, kommt es zu einem Anstieg der Wasserverdunstung über die Hautoberfläche.

\section{Hydratation}

Die Hornschicht enthält verschiedene Substanzen wie Harnstoff und Ceramide, die Wasser binden. Diese natürlichen Feuchthaltesubstanzen tragen wesentlich zum äußeren Bild einer glatten, straffen Haut bei.

Der Wassergehalt der Hornschicht läßt sich indirekt durch Messung der elektrischen Kapazität, die im wesentlichen von Wasser beeinflußt wird, ermitteln. Dafür wird ein Meßkopf mit einem Kondensator auf die Haut aufgelegt und die Kapazitätserhöhung gemessen. Ein Stromfluß findet dabei nicht statt, es wird lediglich ein elektrisches Feld aufgebaut $[2,9]$.

Verminderungen des Wassergehaltes, beispielsweise bei älteren Menschen und übermäßigem Gebrauch von Hautreinigungsprodukten, führen zu einem faltigeren, schlaffen Aussehen der Haut, während andererseits Kosmetika positive Wirkungen durch Erhöhung der Wasserbindungskapazität erzielen.

Daher ist die Messung der Hydratation sowohl zur Ermittlung der Verträglichkeit als auch zum Wirksamkeitsnachweis von Externa von Interesse. 


\section{pH-Wert}

Die Haut hat einen sauren pH-Wert um 5,5. Dieser Säureschutzmantel dient der Abwehr von Mikroorganismen, die ein alkalisches Milieu bevorzugen, und stellt eine Pufferkapazität für chemische Substanzen dar. Kontakt mit alkalischen Substanzen, insbesondere mit Seifen, kann zu einer Störung dieser Schutzfunktion führen [3].

Für Hautmessungen werden Einstabmeßketten eingesetzt, die die aktive und die Referenzelektrode in einem Aufbau enthalten. Die potentiometrische $\mathrm{pH}$-Messung nutzt elektrochemische Grenzflächenprozesse zwischen Glas oder Metall und Salzlösungen. Ein Ionenfluß führt zu Spannungsdifferenzen zwischen den beiden Elektroden, die in den $\mathrm{pH}$-Wert umgerechnet werden können [10].

\section{Messungen der Hautstruktur}

Invasive Methoden an Hautbiopsien gelten weiterhin als Goldstandard der morphologischen Diagnostik. Dazu gehören lichtmikroskopische (histologische, immunhistologische) und elektronenmikroskopische Untersuchungen, die neben einer hervorragenden Auflösung struktureller Elemente auch Informationen über funktionelle Parameter wie Proliferation und Differenzierung liefern. Wie bereits anfangs erwähnt, haben diese Methoden den entscheidenden Nachteil, invasiv und somit nicht beliebig wiederholbar zu sein.

Die Entwicklung nichtinvasiver Methoden ermöglicht eine In-vivo-Untersuchung der Hautoberfläche, der Epidermis und der Dermis ohne diese Nachteile. Zu diesen neueren Meßverfahren gehört die Auflichtmikroskopie, mit der die Hautoberfläche und die Epidermis bis zum oberen Corium horizontal betrachtet werden können. Sie eignet sich allerdings nicht zur Quantifizierung. Andere Methoden wie die konfokale Mikroskopie und die Magnetresonanztomographie der Haut bieten vielversprechende Perspektiven, befinden sich aber z.Z. noch im experimentellen Stadium und sind sehr aufwendig.

Einige ausgewählte morphologische Meßverfahren werden im folgenden beschrieben. Die Profilometrie und die Sonographie sind bereits etabliert, die optische Kohärenztomographie wird als neues Verfahren vorgestellt.

\section{Laserprofilometrie}

Die Rauhigkeit der Hautoberfläche wird von unterschiedlich dimensionierten Falten und vom feinen Oberflächenrelief determiniert. Sie läßt sich mittels profilometrischer Meßverfahren quantifizieren. Die Laserprofilometrie arbeitet nach einem Autofokusprinzip. Ein Laserstrahl wird auf die Oberfläche fokussiert. Jede Höhenveränderung der Oberfläche führt zu einer Defokussierung, die über Fotodioden registriert wird und zu einer Nachregelung der Linse führt. Die Linsenbewegungen werden aufgezeichnet und repräsentieren ein genaues Abbild der Oberfläche, die dreidimensional auf einem Bildschirm rekonstruiert werden kann. An diesem Profil sind Berechnungen der Rauhigkeitsparameter nach DIN (Abb. 1) und mathematische Prozeduren wie die Fourier-Transformation möglich, die über Leistungsspektren und Autokorrelationsfunktionen zusätzliche Informationen über die Verteilung der Frequenzen und Amplituden und damit über die Regularität der Falten liefern [7].

Da die Methode mit einer sehr hohen Auflösung um $1 \mu \mathrm{m}$ arbeitet, ist eine In-vivo- 


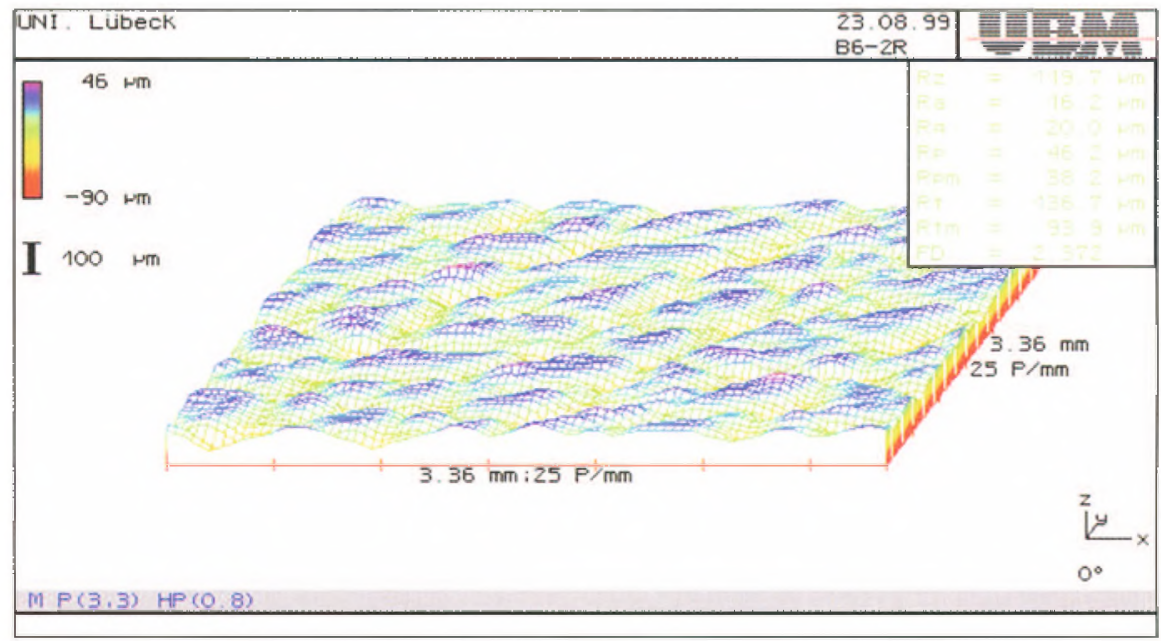

Abb. 1. Laserprofilometrie gesunder Haut von der Unterarminnenseite mit Berechnung der Rauhigkeitsparameter nach DIN. 3,36 mm x 3,36 mm, Auflösung 25 Punkte/mm

Messung aufgrund der feinen Bewegungen durch Atmung und Herzschlag nicht möglich. Deshalb werden Silikonabdrücke der Testareale angefertigt, die dann vermessen werden können.

Chronisch entzündlich veränderte Hautareale zeichnen sich durch eine erhöhte Rauhigkeit mit tieferen Falten aus. Hautpflegeprodukte können hingegen zu einer Glättung der Oberfläche führen.

\section{Sonographie}

Die Dermis und das subkutane Fettgewebe können mittels hochfrequenter Sonographie dargestellt werden. Hautultraschallgeräte arbeiten mit einer Frequenz von 20 $\mathrm{MHz}$. Die Auflösung liegt zwischen 80 und $200 \mu \mathrm{m}$ bei einer Eindringtiefe um $6 \mathrm{~mm}$. Akustische Signale werden an Inhomogenitäten im Gewebe, insbesondere an Grenzflächen, reflektiert, wodurch strukturelle Tiefeninformationen gewonnen werden [ 1 , 5]. Die Dermis stellt sich echoreich, die Subkutis echoarm dar. Dabei interessiert insbesondere die Dicke der Dermis, beispielsweise bei Untersuchungen der atrophierenden Wirkung von Kortikosteroiden. Entzündungsreaktionen und degenerative Veränderungen des Kollagens, z. B. aktinische Elastosen, stellen sich als echoarme Regionen dar. Die Grenzzone zwischen Dermis und Subkutis zeigt bei Zellulite einen wellenförmigen Verlauf (Abb. 2). Die Epidermis läßt sich mit den kommerziell erhältlichen Geräten nicht weiter differenzieren.

\section{Optische Kohärenztomographie}

Die Hautoberfläche kann mittels Profilometrie, Dermis und Subkutis können durch Ultraschall dargestellt werden. Nicht-invasive morphologische Verfahren zur Untersuchung von Hornschicht und Epidermis standen bisher nicht zur Verfügung. Diese diagnostische Lücke kann durch das neue Verfahren der optischen Kohärenztomographie geschlossen werden. 


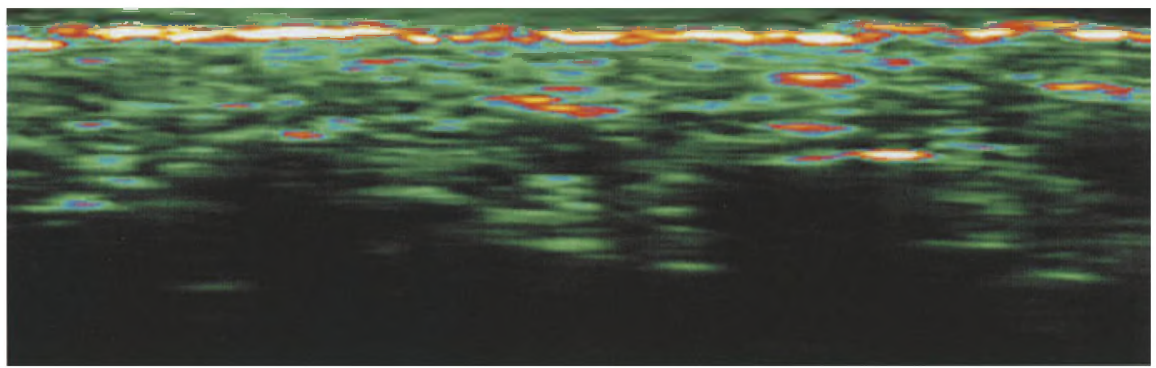

Abb. 2. Hochfrequente Sonographie von Zellulite am Oberschenkel. Die Grenze zwischen echoreicher Dermis und echoarmer Subkutis ist unregelmäßig und wellenförmig. $12 \mathrm{~mm}$ x $6 \mathrm{~mm}$

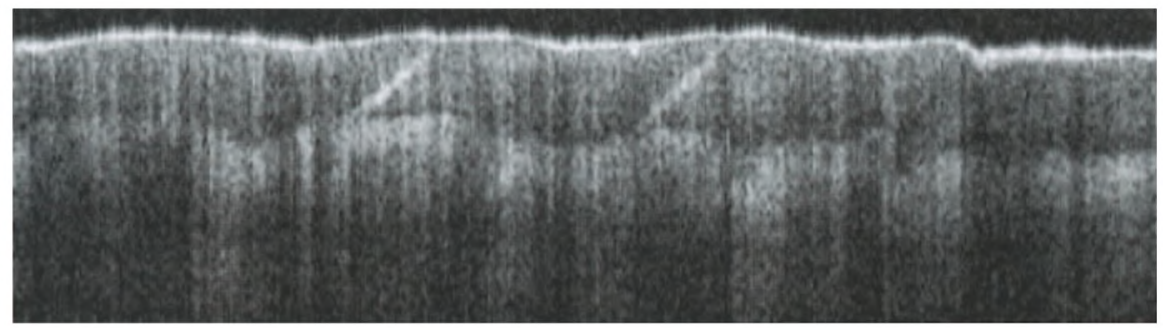

Abb. 3. Optische Kohärenztomographie gesunder Haut am Daumen. Die oberste, signalärmere Schicht ist das Stratum corneum, in dem sich ein spiralförmiger Schweißdrüsenausführungsgang abgrenzen läßt. Darunter liegt die signalreichere Epidermis. $1 \mathrm{~mm}$ x 1,5 mm

Die Methode basiert auf der Michelson-Interferometrie. Infrarotes Licht einer Wellenlänge von $830 \mathrm{~nm}$ wird in einen Referenz- und einen Probenstrahl aufgeteilt. Der aus dem Gewebe reflektierte Probenstrahl kann nur dann mit dem Referenzstrahl, der eine bekannte Weglänge zurückgelegt hat, interferieren, wenn sich beide Strahlen innerhalb der Kohärenzlänge der Lichtquelle treffen, wodurch Informationen über Inhomogenitäten innerhalb der Probe gewonnen werden. Die Kohärenzlänge ist mit $15 \mu \mathrm{m}$ sehr kurz und bedingt die hohe Auflösung, die eine Differenzierung von Zellaggregationen und -schichten erlaubt. Durch laterales Scannen werden zweidimensionale Bilder dargestellt, die mit Ultraschallbildern vergleichbar sind, jedoch optische und nicht akustische Phänomene wiedergeben (Abb. 3). Die laterale und die axiale Auflösung beträgt $15 \mu \mathrm{m}$, die Eindringtiefe liegt bei ca. $1 \mathrm{~mm}[8,12]$.

Die optische Kohärenztomographie kann neben der Diagnostik oberflächlicher Hauttumoren dazu dienen, Veränderungen der Hornschicht und der Epidermis sichtbar zu machen. Einsatzmöglichkeiten dieses neuen Verfahrens sind beispielsweise Untersuchungen von Therapieeffekten, Akanthose oder Atrophie der Epidermis, Hornschichtquellung und Wundheilung. 


\section{Literatur}

1. Altmeyer P, Hoffmann K, Stücker M, Goertz S, el-Gammal S (1992) General phenomena of ultrasound in dermatology. In: Altmeyer P, el-Gammal S, Hoffmann K (eds) Ultrasound in dermatology. Springer, Berlin Heidelberg New York Tokyo, pp 55-79

2. Barel AO, Clarys P (1995) Measurement of epidermal capacitance. In: Serup J, Jemec GBE (eds) Handbook of noninvasive methods and the skin. CRC, Boca Raton Ann Arbor London Tokyo, pp 165-170

3. Braun-Falco O, Korting HC (1986) Der normale pH-Wert der menschlichen Haut. Hautarzt 37: 126129

4. Distante F, Berardesca E (1995) Transepidermal water loss. In: Berardesca E, Elsner P, Wilhelm KP, Maibach HI (eds) Bioengineering of the skin: Methods and instrumentation. CRC, Boca Raton New York London Tokyo, pp 1-4

5. Hoffmann K, el-Gammal S, Altmeyer P (1990) B-scan-Sonographie in der Dermatologie. Hautarzt 41: 7-16

6. Pinnagoda J, Tupker RA, Agner T, Serup J (1990) Guidelines for transepidermal water loss (TEWL) measurement. Contact Dermatitis 22: 164-178

7. Saur R, Schramm U, Steinhoff R, Wolff HH (1991) Strukturanalyse der Hautoberfläche durch computergestützte Laser-Profilometrie. Hautarzt 42: 499-506

8. Schmitt JM, Yadlowski MJ, Bonner RF (1995) Subsurface imaging of living skin with optical coherence microscopy. Dermatology 191: 93-98

9. Triebskorn A, Gloor M (1993) Noninvasive methods for the determination of skin hydration. In: Frosch PJ, Kligman AM (eds) Noninvasive methods for the quantification of skin functions. Springer, Berlin Heidelberg New York Tokyo, pp 42-55

10. Welzel J (1995) pH and ions. In: Berardesca E, Elsner P, Wilhelm KP, Maibach HI (eds) Bioengineering of the skin: Methods and instrumentation. CRC, Boca Raton New York London Tokyo, pp 91-94

11. Welzel J (1997) Profilometrie, Chromametrie, pH-Metrie und sonstige Bioengineering-Verfahren. In: Korting HC, Sterry W (Hrsg) Diagnostische Verfahren in der Dermatologie. Blackwell, Berlin Wien, S 71-77

12. Welzel J, Lankenau E, Birngruber R, Engelhardt R (1997) Optical coherence tomography of the human skin. J Am Acad Dermatol 37: 958-963 